\title{
Managing Quality in Health and Social Care Services; an Exemplary Review of a Center in London
}

\section{Baguma JC ${ }^{1}$ and Obeta $\mathrm{MU}^{2 *}$}

${ }^{1}$ Department of Business and Healthcare Management, Arden University, London ${ }^{2}$ Department of Public Administration and Local Government, University of Nigeria Nsukka, Nigeria

*Corresponding author: Obeta Mark Uchejeso, Department of Public Administration and Local Government, University of Nigeria Nsukka, Nigeria, Tel: +234 8088741364; Email: uchejesoobeta@gmail.com

\section{Review Article}

Volume 3 Issue 2

Received Date: March 16, 2020

Published Date: April 06, 2020

DOI: $10.23880 /$ jqhe- 16000157

\section{Abstract}

Total quality management is day by day showing increasingly high profile of activity and research interest in health and social care. Internal and external stakeholders play their roles to ensure adequate quality care delivery. A care center can use a laid down approved and regulatory standard to improve their services of care. Though challenges such as leadership and management, training, staff and insufficient resources can affect the quality of care, regulatory bodies like NICE and CQC can make health and social care centers to achieve a desired quality service in London. It is therefore, imperative and adequate to carry out internal or external quality assessment of care centers regularly to aid improving the system and quality of care for clients' utmost satisfaction.

Keywords: Quality Management; Health and Social Care; Quality Assessment; Satisfaction; Care Center

Abbreviations: HSC: Health and Social Care: NMC: Nursing and Midwifery Council; NICE: The National Institute for Health and Care Excellence; SCIE: The Social Care Institute for Excellence; CQC: The Care Quality Commission; TQM: Total Quality Management; QMPS: Quality Management Principles; QFD: Quality Function Deployment; QMS: Quality Management System; PIR: Provider Information Return.

\section{Introduction}

The Quality is an increasingly high-profile activity in health and social care, and an essential component within service delivery, due to raised series of concerns of poor performance in health and social care (HSC) [1]. Therefore, the Health and Social Act 2012 requires all health care providers and managers to be more accountable while delivering services in Health and Social Care settings. A human resource and quality manager of any health and social care home, has the responsibility to improve the services. This paper shall demonstrate the understanding of quality management and managing quality in HSC, assessing and monitoring the quality, and safety procedures, whilst explaining the systems, policies and actions that can be taken to tackle any identified shortfalls. A manager should show the understanding of change in management, and the process in implementing Total Quality Management principles, the policies and procedures, quality statements, and managing a continuous quality improvement.

\section{The Stakeholders Perspectives in Health and Social Care (HSC) about Quality}

Quality of care is a process of changing and improving service that meet high standards, and responsive to deliver appropriate services to users, that enable participation in the society and inclusion of users, so that choice is informed, and desired outcome [2]. Stakeholders are group of people, and institutions, who has an interest in any organization's business, performance and its resources [3]. There are two types of stakeholders, 'Internal' stakeholders, such as, service users, patients, and, external stakeholders, such as, employees, care provider, or local authorities. Perspectives 
are ideas adopted by stakeholders, such as, patient, healthcare workers, manager, organization, of which, each have their own perspective, on judging, and deciding, ways of acquiring and improving quality healthcare [4].

Care organizations are, to experience, effective, and efficient delivery of a high-quality service to service users, and staff who present quality performance, committed and competent, in putting more effort to acquire, knowledge, and updated skills [5]. In a HSC center where, staff worked less effectively with external stakeholder, and showed fewer caring responses to users with challenging behavior. Therefore, the center, expect staff to update their training, meet agreed working standards, that are zero prevent breach of regulations, that the Home experienced, such as, a breach of medication administration, and the center, expect both users, and staff to use resources fairly, and comply with legislations, whilst respecting user's choice, and preferences [6].

Employee's perspectives are essential facilities, and adequate infrastructure, that is vital to the sustainability of, and key attribute, to enhanced quality of care delivery, and quality improvement [7]. The staff in a center wish to have a stable leadership, and management support, then, with recognition, to lift their morale. Further, expectation, are to access affordable training, for career development, related to their roles, and individual's care plans, as well as organization structure/culture [8] For example, a staff of a center that lacked some skills, and guidance, in this case, their expectations are, a management support, flexibility, and guidance, as well as, access policies, and procedures, that makes real improvement, with zero defects [9].

Perspectives of service users, or patients, are key to quality care improvement, and it is the quality of the users that matters, thus, their expectations are, to have a quality care that fit for purpose and to be cared with sympathy, humanity, respect, and dignity [10]. Furthermore, they expect user-centres care that lives in a safe environment, with quality services, that are tailored, to their needs, choice, and preferences [11]. The service user, in a care center is expected to be, supported by a knowledgeable worker, who has advanced skills, long-term experience, and good reputation. Finally, service users expect stakeholders to put more effort, in delivering quality care, and their perspectives to be met expeditiously.

\section{Analysis of the Role of External Agencies in HSC Setting Standards, an Instance of London}

The External agencies are, regulatory, independent, and advisory bodies, or commissions, who work in partnership aiming at one goal, which is delivering quality efficiency and effective services in healthcare sectors, both at National, International, and Local level [12]. These, external agencies, can be, "The Nursing and Midwifery Council (NMC); The National Institute for Health and Care Excellence (NICE); The Social Care Institute for Excellence (SCIE) and The Care Quality Commission" (CQC). The role of CQC, is to register and regulates all health providers and adult social care providers in England independently. Therefore, it is primary role is to rate all adult care services provided by Hospitals, Dentists, Nursing Homes, Care Agencies, and Private homes, to ensure they comply with the quality and safety standards. This is done by expert inspections through registration of licensing services that meet its set standards, and use intelligent monitoring, and judgment whether the quality and safety standards are met, and if indicated by the inspection outcome is negative, action is taken and after the results are published for public view. For example, on 12th and 17th December 2018, CQC carried out an inspection, in a Care Home, and found a lot of short fallings, due to breach of Its 16 agreed standards. Therefore, $\mathrm{CQC}$ has a responsibility to respond to any concerns about poor standards over its regulated services, and it is liable for enforcing the law if multiple failures happen again or near the organisations, in this situation.

The role of NICE, is to provide, guidance, based on the best available up-to-date evidence, of which is used to develop recommendations, and advice, that help the public health, health and social care professionals to make decision, on critical assessment, the best possible care is delivered within the resources available. According to the case study, the center provides care services for people with learning disability, and behavior that challenges. Hence, NICE, guidance, and guideline helps the Home, to support the service user, in order to have a quality life that promotes independence, whilst using a lifelong approach, and focusing on NICE products such as, pathways, guidance, quality standards, advice, and shared learning, and published information, via NICE website [13].

The role SCIE, it to identify key types of research, experience and knowledge that combine into the foundation of the social support network and how to develop the quality of social care standards. The agency provides support and services to individuals, families' and children's, to obtain a quality of life. Therefore, they collaborate closely with, other external and inter agencies, publish knowledgeable information, that contain a wide range of resources, links, and other networking, to support those in frontline practice, to improve outcome for service users. The agency laces culture and society at the forefront in identifying the main features of a social care, related learning organization for both the users and the caregivers. For example, in the center, SCIE's resources, provides flexible e-learning, through social 
TV, magazines, and digital library that can help employees, to keep up with up-dated knowledge, about main issues in social care, through guidance and practical tools. Therefore, these external agencies aim at maintaining the standards that help to measure the quality, fit for purpose of provided services.

\section{The Impact of Poor Service Quality on Health and Social Care Stakeholders}

The quality of care is an important factor that increases user's satisfaction, and it's not informed by service users alone, but it is everyone's role, including those who have interest in the matter of quality [1]. However, a high-quality care can be critical for achieving, the desired goals Therefore, it is vital that everyone understands the experience of both good service, and poor services, what high-quality care looks like, and what to expect when using care and support. The Impacts of poor quality on different stakeholders, makes managers tend to feel that access; effectiveness, technical competence, and efficiency are the most important dimensions of quality. However, the services cannot be judged to be good quality, just because it is safe, when its effectiveness or user's experiences are ignored. Therefore, the impact of poor quality in organisations is insufficient attention, critics; failure to identify whether the practice has met the standards or exceeded without damaging organizational reputation [9].

Further, impacts of poor-quality is resource availability, which can influence performance, and disrupt funding can be a result of inappropriate budget planning. Both Newton, et al. [8], suggested that responsive budgeting is another issue that deserves attention as a way to secure resources to end. Both Tibbs, et al. [14] quoted that, poor communication is believed to be the main cause of human errors in clinical settings along with team work, Hence, communication strategies are essential as it engage stakeholders in delivering quality, and service developments, including the sharing of information within themselves, that account for their effort, satisfaction at work and their degree of commitment in delivering quality services [15].

The main underlying quality issues causing negative impact at a given health and care home, is poor leadership, of which should be regarded as the ability to inspire users, and organizational excellence. For example, one of the CQC findings, in the center, was a lack which poses urgent need to update the leadership and management [16]. Facilitating care in the best interest of the service users, is vital, however, based to the case study, residents lacked maximum choice and control of their lives, of which caused decline in patient care, poor services delivered and poor provision of patientcentered care practices [12].
In this case, the impact of poor quality, can lead a number of regrets, such as, loss for value of money spent in care services, and quality improvement. Hence, in such situation, is important that the management to focus on maximizing funds utilization and make effective and efficient arrangements for quality improvement [17-19]

The benefit of monitoring identifies underlying quality issues that cause poor services. For example, some of the significant aspects, in the Care Home, were poor reporting process, and non-compliance that impacted the services, especially when the management failed to notify CQC, of incidents that occur at the service (Regulation 18 Registration HSCA 2014). Hence, CQC inspection monitoring, improves reporting and recording process, by ensuring quality concerns are dealt with on time, and effectively through quality assessment, and, quality assurance, within quality circles. Aim is to ensure that both the perspectives of patients, staff, and stakeholders are given due consideration.

\section{The Standards that Exist in the Health and Social Care, for Measuring Quality}

Standard is described as a document pointing out organisations laws or rules and regulations to safeguard service users and guarantee that service providers comply with laws and regulations. The purpose of standards is to ensure that there is a high quality of service delivered and quality products that can obtain a greater health services that add value on staff performance, individual's needs, and standards, such as, CQC, and NICE standards, or Codes of practice. For example, The CQC (2015) Fundamental Standards, which replaces the existing Essential Standards of Quality and Care, its new inspection, focus on a patient is centered on practice, rather than inspecting a compliance with the regulations, of which the enquiry look at a good care and assesses, whether the practice is safe, effective, caring, responsive and well led (Figures $1 \& 2$ ).

\begin{tabular}{|c|c|}
\hline Safe & $\begin{array}{l}\text { By safe, we mean that people are protected from abuse and } \\
\text { avoidable harm. }\end{array}$ \\
\hline Effective & $\begin{array}{l}\text { By effective, we mean that people's care, treatment and } \\
\text { support achieves good outcomes, promotes a good qually } \\
\text { of life and is based on the best avalable evidence. }\end{array}$ \\
\hline Caring & $\begin{array}{l}\text { By caring, we mean that staff involve and treat people with } \\
\text { compassion, kindness, dignity and respect. }\end{array}$ \\
\hline Responsive & $\begin{array}{l}\text { By responsive, we mean that services are organised so that } \\
\text { they meet people's needs. }\end{array}$ \\
\hline Wellled & $\begin{array}{l}\text { By welled, we mean that the leadership, management and } \\
\text { governance of the organisation assures the delivery of high- } \\
\text { quality person-centred care, supports learning and } \\
\text { innovation, and promotes an open and fair culture. }\end{array}$ \\
\hline
\end{tabular}




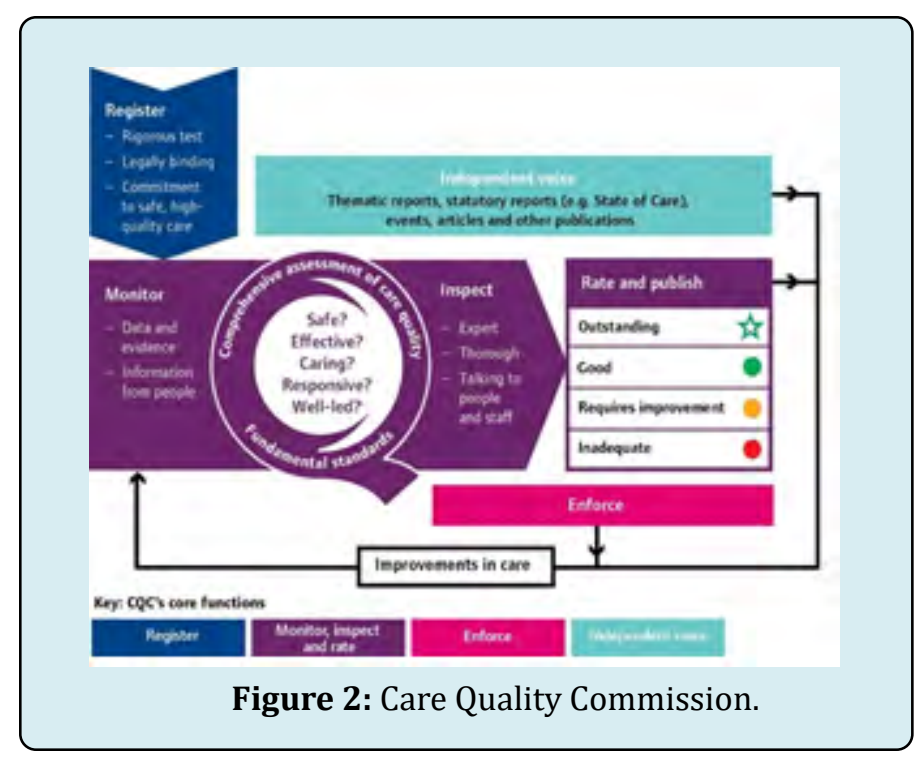

The CQC standards, are under HCS Act 2008, and CQC Regulations 2009, to rate its registered care provider, whether or not the standards have been met or reached. The process, involve, quality control, and quality assurance activities, to promote best possible care, of which team uses professional quality approach, that focuses on inputs, the outcomes, of which their quality measuring creates a zero defects team. For example, the inspectors, inspected the center, assessed and monitored the services against performance, and checked documents, audit, and feedback using statistical quality control, and systematically gathered information, to determine whether the services meet agreed standards (Figure 2) [20]. However, in the center, CQC standards were not met due to short fallings of non-compliance of staff training, governance, medicine administration, staffing standards, and many more.

The NICE of quality standards are in form of specific statements that provides evidence-based guidelines to improve and shape high quality adult social care, locally and nationally. The aim of the guidelines is to support the provision of care, which has shown to work as indicators of high quality cost-effective in delivering the care that is tailored to users and fit for purpose. Even though NICE guidelines are not mandatory, but they are still useful at indicating priority areas, which constitute criteria for quality maintenance in medicines, and treatment. This includes measuring and monitoring quality standards by CQC [21].

However, CQC report in the center shows that the care services were not delivered in line with evidence-based guidelines, meaning the NICE quality standards, were not effective. In this case, quality improvement, can identify the gap, and the center output, and Input process is adherence to NICE requirements, see Figure 3, or do contribute to the quality and the safety of everyone.

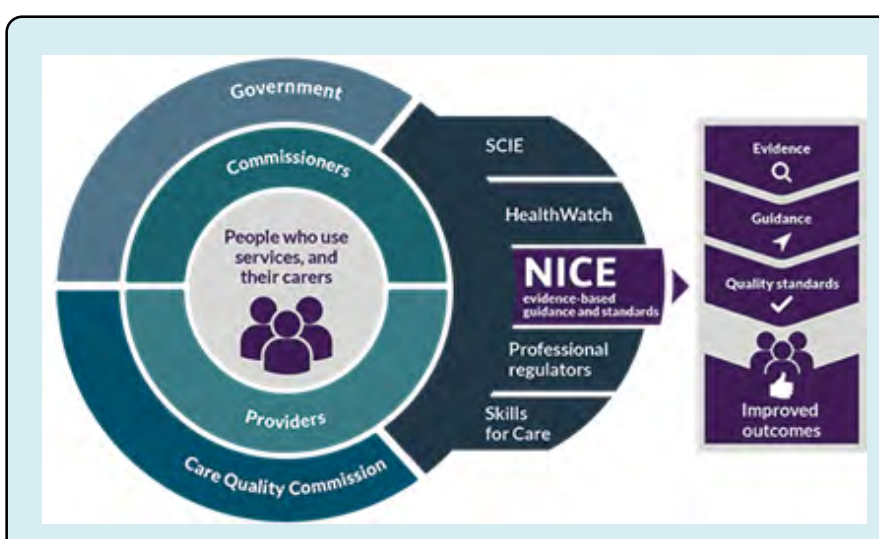

Figures 3: The NICE Quality Standard in Adult Social Care. Source: (NICE, 2019) [21].

The Code of Practice, of Social Workers, standards, interprets a proof of good and wrong performance, and how to achieve quality standards. For example, it states that workers must be accountable for the quality of their work, have a responsibility of maintaining and improving their knowledge and skills. This includes, reflects and explains how workers should behave in front of service users, and describes the highest standards of professional practice, and the protection of the vulnerable users. According to the case study, the center, staff performed poorly, and responded inappropriately, of which was a non-caring way, particularly to the residents who were challenging [21]. The Code of Practice standards, expect staff in the center to perform whilst continuously complying with the six key roles, and ensure their training is updated. Meaning that the care home should have a clear standard that guides them, whether they have achieved the necessary results.

\section{Different Approaches to Implementing Quality Systems}

A Quality Systems are organisation's outlined policy and procedures that monitors all the activities and tasks, and check whether the quality management, is on track, or not, or the services meet customer's needs, and provides value products. However, they are different tools to maintain the desired level of quality system to excellence in the organisation, which includes: Total Quality Management; Continuous Quality Improvement, and Benchmarking [4].

Total quality management (TQM), is the act of achieving a benchmark level of quality through formulating a quality policy, planning, assurance and quality control for quality improvement. It consists of new edition of ISO 9001:2015 standards based on seven Quality Management Principles 
(QMPs) (Figure 4), which are fundamental beliefs, norms, rules and values that are approved to be implemented to guide an organization's performance improvement or applied as a basis for quality management [16].

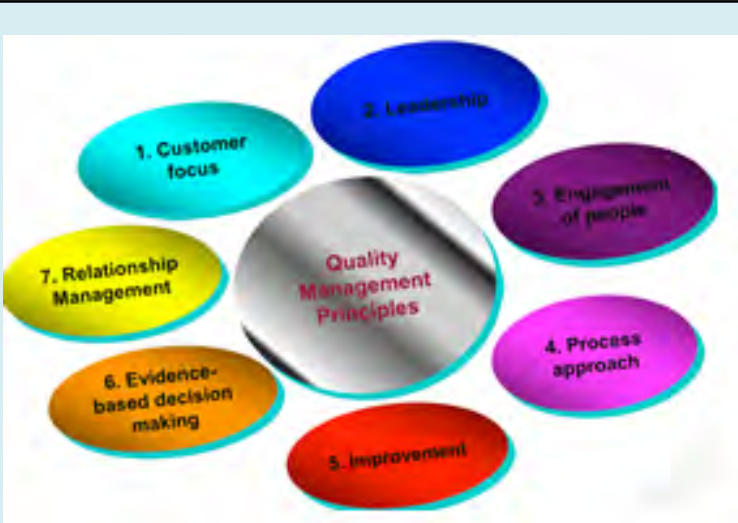

Figure 4: Illustrates seven principles of TQMP. Source: (ISO, 2015).

Focused on customers' needs, every organizations depend on the customer [22] therefore it is responsive to meet customer's current and future needs, and to strive to exceed their expectations by delivering the consistency level of quality service beyond their expected levels of satisfaction [23]. However, meeting customer's needs places an emphasis on Quality Function Deployment (QFD), to help in learning, the customer's requirements, and to measure their satisfaction. Hence, customer feedbacks and expectations need to be carefully monitored and evaluated to deliver superior quality products, as this will increase customer's value, enhance their loyalty, and market share especially in medical laboratory services [4].

The leadership has a commitment of setting clear goals, and to establish unanimity of purpose by involving subordinates to work towards agreed goals, and standards. Hence, leaders create conditions which people are engaged within achieving the organization's quality objectives and set direction that can result into effective long-term quality performance of organization. The maintenance leader has to be self-motivated, organized, trustworthy, understanding and optimistic. In other words, a leader establishes a clear vision of the organization's future, set challenging goals and targets, and provide resources, conducive environment, and essential guidelines, to sustain shared values, fairness and ethical role models at all levels of the organization.

The Involvement of people requires everyone in the organisation to be competent, empowered and engaged in delivering services that create value (Figure 5). For example, employee's recognition, enhance their skills, and capability of producing quality services, and their abilities can help in planning future goals, increase their motivation to achieve organization's goals, or objectives [22]. Hence, people's involvement at all levels, can enhance a sense of own well-being, and to be part of organization's success, and customer's satisfaction [24].

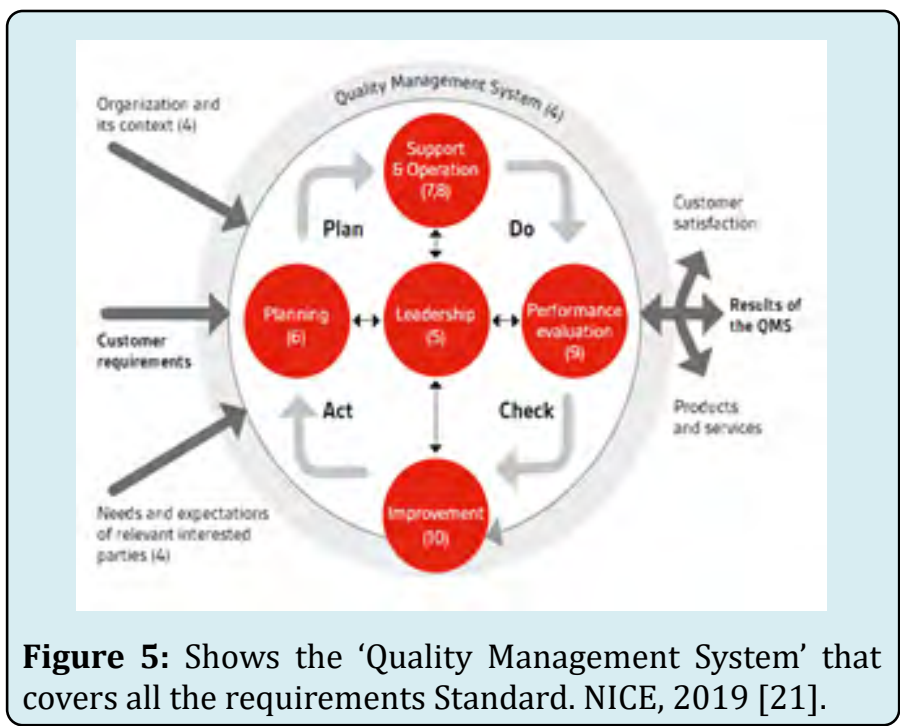

However, in the center, 'Process approach' would be best approach, to achieve its objectives, because this principle is a foundation of developing Quality Management System, (QMS) in every organisations per as the ISO 9001 Standard, of which promotes the effectiveness and efficiency of a quality management system operation, that enhance customer's satisfaction.

The advantage of TQM principles increases the reputation of the organisations, of which attract more customer, by enabling participative in decision making, and involvement of people at all levels. Further, the continuous quality communication between levels and functions of the organisations, increase capability of meeting customer's expectations and delivering the desired results. In TQM, help in quality improvement in healthcare system performance, of which can ultimately suppress errors, and result in achieving the high-quality care [25].

The disadvantage is, that their extent of participation, can vary from advising to power-sharing, and ensuring that customers are given choice and control when planning their care, and whether that care is based on individual needs, wishes to strive and exceed customer expectations. Therefore, to achieve the organisations success, the seven principles of TQM, should be properly implemented, even though they have a significant implication, they need to be considered. 
Benchmarking is the study, against the competitions of making a comparison between two businesses, the planned practices or comparable activity, to determine how well other organizations are doing, by identifying their best performance. Hence, Benchmarking is based on the findings, study is from one company, on another organisation's performances both internal and external (Figure 6), to compare between similar departments or organisations for the purpose of identifying if the practices or generating ideas can be, adapted, by the particular services doing the study, in the aim to improve their own services [23].

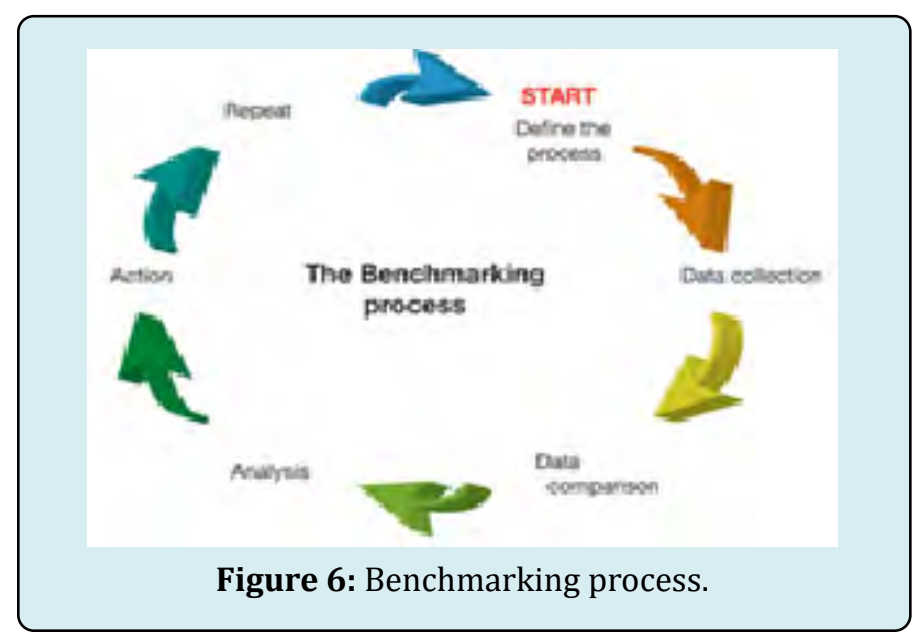

The Advantages of Benchmarking provides a better understanding of competitions and enables the organisations doing the study to focus on the processes that are critical to its business, and measure those that are most relevant, and specific attributes of its own products, services, practices, and other activities. Further, benchmarking enables rapid changes help the organisations to identify its strength and weakness, performance gaps, and to improve its own performance, weakness. It gives a new idea to establish how other companies achieved their superior performance level and the costs of what is being benchmarking.

The disadvantages, the benchmark does not benefit every organisation, as it applies on organisation that are of same level of standard. For example, Rose Meadow Care Home, cannot benchmark with NHS Hospitals, or St Patrick cannot bench mark with Cambridge University and so on. Finally, there is a risk of breaching confidentiality, in the process of knowledge being shared with different competitors and a compromised benchmarking can also be harder to meet a criteria.

\section{Potential Barriers to Delivery of Quality in Health and Social Care Services}

Barrier is anything that hinders the progress of an individual or gaining access to services or prevent organisation to achieve its goals or to do the right things. In regard to HSC sectors, one of the potential barriers in care setting is lack of leadership, effective communication, training, and resources.

Lack of leadership- every leader, should focus on the processes that are critical in organisation, implement policies, shared vision for improvement, quality, set long term goals, guidelines, ensure the safety of the people, and communicate issues. However, in the center, CQC reported that, there was no registered manager in place, and for six months, the services had no manager, but a newly recruited manager was in the process of registering. Therefore, lack of strong leadership, led the services not to meet the legal requirements, and CQC standards that regulate the services, of which also impacted staff performance in providing high quality care, in meeting service user's needs, and increased organization's reputation [26].

Lack of training- Training is a major factor for any organisation; hence it is essential to invest in continuous training development, and quality awareness, to adopt TQM successfully, because training can also make employees' usage of TQM tools effectively. However, the implementation of quality system can only be achieved through training, and certain level of academic skills about quality. According to CQC report, 16 Staff in the center, had insufficient training, of which led the organisation to face various breach of the HSCA 18 Staffing Regulations.

Meaning some staff was not conversant with delivering care in specific areas, and to perform up to the standards that meets customer's expectations. Hence, lack of skill deficits, can also result into perceived threat and negative attitudes, of unprepared to perform specific tasks. However, the process of investing in training, and implementing change, in the care center, also needs skills of change management, however, the plan can be affected by financial implications, and a considerable resource dedicated from budgets constraints that are always under great pressure.

Lack of resources, and products to facilitate organisation structure, can result to obtainability of inappropriate facilities to use, and facilitate the training, services, and change, of which may not benefit the service user, and the staff.

Further, insufficient resources, can lead to poor maintenance of technical system support, may cause staff resistance to changes, and increase patient's satisfaction, or impact service delivery. According to CQC, the new manager had begun to implement longer term goals, but was not effective, and staff training was not updated. Hence, 
inappropriate training, and having unrealistic goals, can result into frustration, as well ultimate rejection of using resources appropriately, and can lead threat to personal adaptation of TQM tools. In this case, the manager has to identify the key barriers to successful change in quality improvement, and investigate how the new system can enhance the delivery of quality service, and procuring the latest resources, to ensure there are effective TQM tools to address the needs of individuals (Department of Health, 2008).

\section{Effectiveness of Systems, Policies and Procedures used in a Health and Social Care Setting in Achieving Quality in the Service(s)}

Systems are designed frameworks, that involves any activities or project that provides quick access to support services users, or customers and to access from, innovative stakeholders, at the leading edge of healthcare system reform. Policy, and Procedure, is statements laid down, to place duties for development and maintenance of a high quality care that drive the health workforce, and suggests the way the set goals, objective or operation procedure or activities should be carried by specific people, and achieved [27]. Therefore, systems, policies, and procedures, regulations, are closely interconnected with regulating bodies, and healthcare institutions, to improve practices, and care services, of which involve- Human Resource; NICE Guidelines; CQC Regulations-Person Centered Approach; Medicine Management; Healthy and Safety; Governance, and Safeguarding policy [28].

A person-centered care policy, under Regulation 9 HSCA- Person centres care, its purpose is to ensure the provided care, and treatment to service users, meet their needs effectively, and reflect to their choice, and preferences, as well as their care plan. The policy enables autonomy, involvement, and a long-term service that offers continuous care that puts the preferences and aspirations; culture and dignity of the individual.

Linking to the case study, CQC report states that improvement is required to ensure service user's activities are person centered, as their care plans did not include users input to allow person centered care. Hence, the center manager needs to arrange enough care workers to improve user's ability and motivation, by ensuring service users are at the top of their services that lead active and meaningful lives. For instance, a well-planned recruitment, and sufficient resources can provide a good work force, minimise turnover, workloads, and increase service users' satisfaction [29].

Monitoring System under Regulation 17 HSCA 2008 RA Regulations 2014 (Good Governance) setoutaboutregulation, residually audits, action plans, regular supervision, meeting minutes. The purpose is to systematically collect and analyses information on the progresses of the organisation.

Therefore, the center has a responsibility for quality assurance measurement of governance and monitoring system, to check whether the quality and safety of care, the plans and system meet the standards, and help the service to improve, as well to reduce any risks to people's health, safety and welfare. However, in CQC report the Home had no governance to ensure good outcomes for people of which put users at risks. The home had no clear strategy plan, as a result there was poor oversight of concerns, of which also the audit highlighted about multiple areas of unsafe care, that remained unresolved, and not addressed promptly and effectively [29].

In other words, their monitoring system and audit was unable to observe, track, and record the implemented activities, and the progress of the organization's set goals. Therefore, the center needs effective quality assurance, an effective strategy plan, and Risk management that involve reporting, accident and incidents, or near misses, to guide staff in their practice, and to ensure risks are determined and managed timely in services. This will enable integrated care to prevent anti-competitive, behavioral control, continuity of the quality improvement and maintenance of quality services (NHS Foundation Trust,).

Medicine management system, under Regulation 12 HSCA RA Regulations 2014 Safe care and treatment its purpose is to ensure the safe handling, storage and administration of medicines. However, CQC findings in the center, indicates that, medicines were poorly managed, poorly stored, and some were out of date. The medicines that were prescribed did not have a clear protocol in place to ensure staff knew when to administer them, and those in the Home, were not on people's medication records. In this case, if the medicine that was administered did not indicate the purpose of treatment, of which means the service users received sedative medicines they did not need. Therefore, the Five Principles rules of medication were not followed, and systems were not reviewed to ensure staff managed medicines safely, of which placed the vulnerable users at risks, and the policy to be non-effective [24].

The benchmark of medicine. The Quality Assurance Agency of 2002 requires doctors to be able to work with other health care professionals to give appropriate input to multidisciplinary teams and promote effective inter professional to minimize medication errors [17]. Hence the care centre manager, is required to assign a competent and qualified person to monitor and manage the way medicines were administered, and cross check the medical chart, of each patients / service users, as not complying with five 
principles, can lead to death, loss of Job, and organisation reputation [24]. For example, the Institute of Medicine, suggested, that health care quality is also known as "the degree to which services for individuals and populations increase the likelihood of desired health outcomes and consistent with current professional knowledge. Hence, the employees require medicine training, supervision, and to be offered proper recognition and the rewards for their activities and the services, as this will enhance high quality care and proper services to the service users [30].

\section{Other Factors that Influence the Achievement of Quality in the Health and Social Care Service}

\section{The following are the few other factors that influence the achieving quality in HSC service}

Safe environment and or safe services, provides quality care, and enables quality performance, especially when staff are supported with regular supervision, training, and career development programmers, as well as provided with sufficient resources, of which can promote staff continuity, reduce staff turnover and increases customer's satisfaction, as well as, leads to improved standards of care quality. According to the CQC report, the center had safety concerns about the service and the outcome, as people were at risks due to numerous concerns regarding inadequate services, and complaint that were not responded to, and lack of meeting users' needs effectively. For example, in the center, the records of those who displayed inappropriate behaviors, and at risk of causing harm were not included in safety care plans, and the fire doors that were broken for over a year, no action had been taken to resolve this. The home lacked interventions, to deal with challenging behaviors, as a result staff responded inappropriately and in a non-caring way to such incidents (for example deciding punishments for behavior), meaning responses to challenging incidents were, unskilled and uncaring, due to lack of guidance, and training. Hence, effective Risk assessment relating to environment, Health and Safety, and individual's mental capacity should be reviewed and updated consistently, as well as, ensure the monitoring systems and process for protecting service users at risks operates effectively in order to have a safe environment. For example, the staff should also be responsible in monitoring, recording, and reporting incidents, the wellbeing of individuals, and any allegations or indicators of abuse. The center can also work in partnership with Clinical Commissioning Group, Safeguarding Adults Board partners, and Quality and Care Governance Team Social services, Local Council to ensure the safety of those using the services. The aim is to safeguard the welfare of the vulnerable service users, to ensure they have a safe and stimulating environment in which they can fulfill their potential.

Funding, or high costs: Sufficient resources or enough funds are likely to provide quality services, that benefits both employees, service users and the management, as well as afford to equip staff with skills, and meet user's needs effectively. There is no standard yet for measuring and controlling the costs associated with implementing quality programs, however, CQC inspection, also involve rationale for assessing the use of resources and the Health and Social Care Act 2008 recognizes if there is a relationship between quality of care and the efficient and effective use of resources, as a result the Act requires inspectors to be conscious of providers' use of resources within CQC's overall purpose as a quality regulator.

Factors such as, resource availability can also influence performance, and theses performance indicators sometime pays insufficient attention and critics argue to factors, due to quality control dominated by standards [31]. Further, when there are enough funds, they can help in increasing people's knowledge, and lack of costs can lead to deliver low services, of which can be problematic especially when there is no cooperation due to lack of knowledge VS those with no knowledge. Based on the case study, the poor resources response could be attribution due to lack of not acknowledging, and rewarding their employees for their services, other factors such as increase wages, employment benefits which makes the home competent rather than on a strategy failure [20].

\section{Ways of Improving Quality of Health and Social Care Service}

Based on the CQC findings in the healthcare center, some of the service was inadequate, and some required improvement. The CQC was concerned about inconsistent managerial arrangements, that impacted on the ability of the service to operate safely and smoothly, and poor management of accidents and incidents, placed service users at risk, and no measures were mitigate any risks. 16 staff had expired training and other lacked specific training, whilst system processes and procedure were non- effective. The risk assessments were incomplete, and service users care plans were not updated of which lead the staff to give service users wrong care and treatments [1].

Lack of effective management, and good governance: the center requires a permanent manager, to enable effective governance, and to enhance the monitoring system to check on the quality and safety of care, the plans, that will help the service to improve and meet the standards, as well as to reduce any risks to people's health, safety and welfare [31].

Staff Lacked training: the staff were struggling to meet the service needs of the service users, due to insufficient training of which resulted some users' undignified ways. This 
can be overcome by reviewing organization's recruitment procedures and the training policy and procedure needs to be reviewed, to ensure there is regular supervisions to improve staff training needs, and concerns. In addition, staff requires being active part in mandatory training, attending in-house, online training, external training secessions and other courses, such as NVQ'S, as well other e- learning and development opportunities, in order for them to provide quality services.

Poor systems, and procedures, resulted into inadequate services, and adverse effect on peoples' personal care needs, well-being, staffing level, that resulted also into unmet needs. In the center monitoring and audit. This can be improved with proper staff training and proper documentation and checking meds chart. In this case, the poor systems and procedures resulted into inadequate services, and adverse effect on peoples' personal care needs, well-being, staffing level, that resulted also into unmet needs.

Hence, we can see how staff was struggling to meet the service needs of the service users, for example service users were left in undignified ways. This can be overcome by reviewing their recruitment procedures and recruit more staff.

\section{Methods of Evaluating Health and Social Care Service Quality with Regards to External and Internal Perspective}

Quality Inspection: This is a routine, scheduled in-depth investigation of any or all of the departments which can be done internally or externally. There are many methods which can be applied in the investigation such as supervision, audits, review, questions, findings, observations, and questionnaire, or telephone questionnaire, and Provider Information Return (PIR) forms that asks the provider to gives an insight of the current situation, and some key information about the current service, and what the service does well and improvements they plan to make. For example, prior to the inspection CQC look at the past information they held about the service, to obtain rich and accurate information/data to measure the quality.

The inspectors also collect data of, notifications, daily reports, care plans, medication charts, and clinical records; results from surveyor questionnaire conducted the manager; or feedback from organisations service user's surveyor, data from both departmental, and performance data and many more. For example, the inspectors in the center used the comments from staff, feedback of the services user who used the service, and from other health and social care professionals in line with Henderson, et al. [20]. Notably, CQC spoke with staff and found that 16 staff had expired training, and took time talking to staff, the interim manager, and the regional support manager at the time of the inspection.

Therefore, CQC designed a well-thought out strategy for how to collect rich data, by gathering information in form of face to face group questionnaire/interview, one to one telephone interview/questionnaire, observations both quantitative or qualitative, and verbally or non-verbally, before, during, and after the inspection have been carried out and change implemented. Then, the information gotten was used to analyze the problems faced by the Healthcare center.

Quality Audit method is an in-depth systematic investigation in any area of an organisation, like healthcare organisation. An audit subject each area of the organization's activity to a systematic critical examination, investigates each area of activity, and examines in depth the audits all work done in each area which helps to identify any errors and mistakes or breach of regulations. It is also an unbiased evaluation and examination of organizations financial records which can be done internally by a manager or externally by another organisation. Therefore, it can be suitable in monitoring the center's financial sustainability, as it is an unbiased evaluation and examination of organisations financial records, which is usually done internally by a manager or externally by a firm. The plan can help the care home to budget well in recruitment process, and training, with regards to staffing issues.

Hence, the benefit of the plan is that it can gather accurate information, assist in planning, obtain investment, maintain accounts, facilitate comparisons, amalgamate the organization, detect and prevent errors. The plan will put in place effective and efficient arrangements for quality improvement, quality assessment, and quality assurance to ensure that the perspectives of service users and their care needs are given due consideration [31]. Hence the center's internal Auditors needs to prepare a report of recommendation which emphasis on how improve needs to be implemented, however the quality audit takes time and is costly. However, if the auditor is biased, the recommendation report might not reflect how effective the report will be either positively or negatively

Quality survey method, is detailed, in-depth examination of a narrower field of activity, (Questionnaire) which are usually embedded into the organization's activities, in order to ensure that the process or plan is seamless can also be used in monitoring by gathering information using survey satisfaction questionnaire, from a representative sample of patients, their family members, and staff. For example, the method, involved many participants with, multiple views, or ideas, and opinions, include those who want to remain anonymous, and monitor non-effective services, or 
performance within organisation or if is suitable, because its effectiveness can be assessed by experts, since it occurs within the organisation to determine organisation's success in quality improvement, change or failure [32].

However, in order to carry out such type of survey, a center has to spend its cost, as well as time, as surveys are time-consuming, and co-operation from participants may be difficult to achieve. For example, poor services that occurred within the practice, and impacted on both service users, satisfaction, can be assessed using this method, whereby individuals are asked the same questions in more or less the same way. The survey may be self-assessment type or external assessment in nature.

The advantage is, the method does not to describe the particular individuals who, participated, but obtain a composite profile of the population, include accuracy of information and high data reliability.

However, Quality assurance process can help to audit quality requirements and the results of quality control measurements to ensure appropriate quality standards, such as, input of quality assurance performance, the costs and work performance. Hence, the center can develop a mapping using the well-known concept of quality costs and the Framework Core produced by the National Institute of Standards.

Effective Communication is one of the major factors that can positively influence achievement HSC setting, in this case, effective communication process, should be transparent (verbal, written, visual) within all levels of management, so that to ensure common language of quality, and accurate shared information. For example, according to CQC findings, some service users were unable to communicate clearly, and staff spending time to understand their non-verbal signs. Further, if the center staff did not work effectively with other different organisations to provide effective support, and no service user was assisted to seek an advocate. In this case, working together with other external bodies, such as, Advocates, British Sign language services, Therapist, can help to deliver a quality care, and to meet the needs of those who lack mental capacity, and inability to communicate.

In other words, maintaining effective communication in the center requires a proper communication procedure, to increase the effectiveness and efficiency in meeting the organization's quality objectives, and an improvement in communication between levels and functions of the organisation. In other words, communication skills, and improved relationship between staff, manager and service users can better coordination, develop and improve the capability of entire management to deliver the quality service or desired result.

In addition, a continuous improvement is an essential principle to implement in the center, as it promises customers satisfaction, and continuously involve all personnel consistently to deliver quality that it implies a never-ending effort towards quality improvements of all aspects of an organisation. The Home should use TQM tools, to maintain a continuous improvement and a successful change of its management/ leadership, because the home has a lot to improve with time, and has to get the best results, that meet CQC standards that focus on person-centres services [25].

Hence, to provide quality care, often depends on the cost as the components of quality care demands expense, and the organizations seeking to gain competitive advantage by focusing on managing costs, periods and quality of services or products. However, it is important to use qualified and skilled professionals to keep the high-quality service, as the Healthcare suggest that quality can actually be enhanced when some cost drivers particularly those associated with intra-case utilization management are appropriately minimized and standardized.

In this case, the center, can invest in staff, and build a continuous learning and performance improvement culture, and ensure it is in a better position to design an organizational structure that sustains quality progress towards quality care, and support services. However, there are still a number of factors which are not related to cost in terms of quality of care, such as, the lack of staff training can decrease the service quality, and especially when staff are not clear about their own responsibilities and roles. Further, the center can develop a mapping using the well-known concept of quality costs and the Framework produced by the National Institute of Standards.

\section{The Impact of Involving Users of Services in the Evaluation Process of Service Quality}

The advantage of service user's involvement in service delivery, evaluation, and quality improvement makes them feel respected, increases their autonomy, independence and empowers them as well as encourages users, to share their emotional attachment, views, of which also makes them to feel loved, cared, and recognized. Hence service users should be involved in all stages of their care and, be at the heart of every care, and in the process of review, it is important to strongly involve them, as well as their engagement to be a continuous process. For example, to meet service user's social needs, requires encouraging their involvement, social inclusion, and autonomy, of which increases their social activeness, feel a sense of acceptance, in the society, as well as maximize their self- esteem, and self-actualization needs 
to be met with full potential [33].

Further, some of the advantages are, necessary support can be given, to build their confidence to carry out assigned duties effectively. Additional support, may include promotion of welfare of clients, maintain ethics, standards and give instant support to help service users to assist care givers where necessary which can improve their health wellbeing. Teaching them social life care skills will empower them to live more independent, and access equal opportunities [17].

For example, if the service user knows what they are entitled to, they will be assertive about their services, help them to get information about their treatment history and other outcomes especially when prescribing medication. It is good to involve service users and when they have reaction or complication, they will let their caregivers know. The involvement also provides service user with the knowledge about how the system works and brings expertise with the best idea to provide quality care, and also sharing agenda, of which promotes constructive working relationships. Hence, it is good to ask the service users how they feel overall, and quality become higher quality care, and also sharing agenda, of which promotes constructive working relationships.

According to CQC report in the center the records showed that people had little involvement with their care planning, as a result, further improvement was needed to embed their full involvement into the service, because service user's involvement can also take the organisation in a better position. Service user's involvement in the center can attribute on the insights gained from analyzing and evaluating data, needed to set up new targets for further quality improvements, and to produce desired quality result.

The disadvantages are that service users' needs reassurance, emotional support, and to meet their Intellectual needs, through enjoyment activities and therapies, to encourage their involvement [1]. Further disadvantages of involving service user, can be lack of knowledge to make realistic demand, as a result the unrealistic demands, can increase the disguise. Some of the disadvantages of involving service users is that some of them ask for things that are not important, that are not appropriate to their care. If the service user is lacking capacity in decision making, choice, or to communicate their ideas, the involvement may not be effective, unless the user involve advocate Ballantyne, et al. [34] Advice giving by health care professional, it will affect their treatment in future, and lack of knowledge may affect information giving. Some see service user input as irrelevant, and waste care givers' time, while some of them give false information because of misunderstanding of their situation or shyness.
According to the center in this case study, the involvement can increase the productivity, however, the disadvantages are bias by supervisors that can lead to staff resistance, and when the CQC become baise, the organisation may not get the clear information. The health and social care center, had some good caring staff, however lack of involvement and management support, may have resulted into noncaring services, and service users' dissatisfaction, of, which attributed to failure [34].

Many negative things will be altered by the service users when their needs are not met, some service users may voice their needs, but it will be weighed under the law, though they may feel they are being ignored.

Some strategies are necessary to involve more service users; the organisation must have policy and procedures for developing their staff and the service users, there must be efficient communication between the service users and staff, monitoring is very important by collecting feedback from staff and service users for example feedback from service user about their fears is very important, the fear complain must be treated fairly, the feedback from both sides must be honest and open.

\section{Conclusion}

The Quality Manager needs to understand what drives service user's experiences of their services, and understand information given to the users, in order to regain their trust back. This approach will improve quality in the Care Home, and help to observe the staff attitude towards service users, include performance, through effective teamwork; as a result it will reduce errors, and non-compliance, waiting times and delays in delivering services, which is a sign of good quality services $[35,36]$.

\section{References}

1. Johnson K, William I (2007) Managing uncertainty and change in social work and social care. Russell House Publishing Ltd., pp: 144.

2. DOH (2000) A Quality Strategy for Social Care. London: HMSO.

3. Freeman RE (1984) Strategic management: A stakeholder approach. Pitman series in business and public policy.

4. Obeta MU, Maduka KM, Ofor IB, Ofojekwu NM (2019a) Improving Quality and Cost Diminution in Modern Healthcare Delivery: The Role of the Medical Laboratory Scientists in Nigeria. International Journal of Business and Management Invention 8(3): 8-19. 
5. Blumenthal D (1996) Quality of health care. PART 1: quality of care-what is It? The New England journal of medicine. 335: 891-894.

6. DOH Department of Health (2013) Drug regulations.

7. Leon W, Wylie J (2010) Assessing user perceptions of staff training requirements in the substance use workforce: A review of the literature. Drugs: Education, Prevention and Policy 17(5): 618-631.

8. Newton DC, McGillivray JA (2019) Perspectives of careers of people with intellectual disability accessing general practice: "I'd travel to the ends of the earth for the right person". Journal of Intellectual \& Developmental Disability 44(1): 64-72.

9. Martin V, Henderson ES (2001) Managing in health and social care. London: The Open University.

10. Mullender A, Coulshed V, David NJ (2006) Management in Social Work. In: Practical Social Work Series, $3^{\text {rd }}$ (Edn.), Paperback, pp: 256.

11. Berwick DM (2008) The Science of Improvement. JAMA 299(10): 1182-1184.

12. Vivien M, Charlesworth J, Henderson E (2010) Managing in Health and Social Care. $2^{\text {nd }}$ (Edn.), Abingdon: Routledge, pp: 384.

13. NICE (2011) Clinical Guidelines.

14. Tibbs SM, Moss J (2014) Promoting teamwork and surgical optimization: Combining team steps with a specialty team protocol. Association of Operating Room Nurse Journal 100(5): 477-488.

15. Galletta M, Portoghese I, Coppola RC, Finco G, Campagna M (2016) Nurses well-being in intensive care units: Study of factors promoting team commitment. Nurs Crit Care 21(3): 146-156.

16. Suarez J (1992) Three experts on quality management: Crosby PB, Edwards W, Joseph M, Juran, et al. 92(2): 1-24.

17. Holroyd J, Field R (2012) performance coaching skills for social work. London: sage.

18. Hafford Letchfield S (2010) Social Care Management, Strategy and Business Planning. London.

19. Letchfield HT, Chick NF (2008) Leadership and Management in Health and Social Care. London.

20. Henderson J, Atkinson D (2003) Managing Care in Context. London, The Open University, USA.
21. NICE National Institute for Clinical Excellence (2006) Supporting people with dementia and their carers in health and social care. UK: NICE Clinical Guideline 42.

22. Rusu C, Rusu V, Roncagliolo S, González C (2015) Usability and user experience: what should we care about? International Journal of Information Technologies and Systems Approach 8(2): 1-12.

23. Obeta UM, Goyin LP, Udenze C, Ojo J (2019b) Assessment of Job Satisfaction Indices among Health Professionals in Jos University Teaching Hospital: An Analytical Study." IOSR Journal of Business and Management 21(2): 38-50.

24. Hayes J (2007) The Theory and Practice of Change Management $2^{\text {nd }}(E d n$.$) , Palgrave Macmillan, pp: 592$.

25. Langley GK, Nolan T, Nolan C, Norman, Provost L (1996) The Improvement Guide: Approach to Enhancing Organisation Performance, $2^{\text {nd }}(E d n),. p p: 512$.

26. Trebble TM, Heyworth N, Clarke N, Timothy P, Peter MH (2014) Managing hospital doctors and their practice: what can we learn about human resource management from non-healthcare organisations? BMC Health Serv Res 14: 566.

27. Hollnagel E (2014) Safety-I and safety-II: The past and future of safety management. Farnham, Surrey, England ; Burlington, Vermont: Ashgate, pp: 198.

28. Gurusamy K, Aggarwal R, Palanivelu L, Davidson BR (2008) Systematic review of randomized controlled trials on the effectiveness of virtual reality training for laparoscopic surgery. Br J Surg 95(9): 1088-10897.

29. Adair J (1993) Effective Leadership. London: Pan Books.

30. Kerem Y, Cevik H, Șimșek IY (2017) The evaluating of service quality in recreational sport events: kite festival sample. PJSS 8(1): 73-93.

31. Field R, Brown K (2010) Effective leadership, management, and supervision in health and social care. London, USA.

32. Schiavo R (2013) Health Communication: From Theory to Practice. $2^{\text {nd }}$ (Edn.), San Francisco: Jossey-Bass, an imprint of Wiley, pp: 656.

33. Uchejeso O, Mabel O, Longul G, James O (2019c) Abraham Maslow's Hierarchy of Needs Dynamics in the Administration of Public Organizations under the President Buhari"s Democratic Dispensation in Nigerian Health Sector. 3(1): 284-290.

34. Ballantyne I (2009) Recruiting and selecting staff 
inorganizations. In: Gilmore $\mathrm{S}$, Williams $\mathrm{S}$, et al. (Eds.), Human Resource Management, Oxford: Oxford University Press, pp: 89-110.

35. Charlesworth (2003) Making a sense of change management. $2^{\text {nd }}$ (Edn.), In: Seden, Janet, Cameron, Green.

36. DOH Department of Health (2000). Health and Social care.

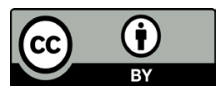

